\title{
Metal Insulator Transition in Vanadium Dioxide Hydrated by Means of the Plasma-Immersion Ion Implantation Method
}

\author{
A Pergament*, S Burdyukh, 0 Berezina and P Boriskov \\ Petrozavodsk State University, Russia
}

*Corresponding author: Pergament A, Institute of Physics and Technology,

Petrozavodsk State University, Petrozavodsk 185910, Russia.

\begin{abstract}
In the work, we explore the modification of the structure of vanadium dioxide films, as well as the metal-semiconductor phase transition in them, when hydrated by the method of plasma-immersion ion implantation. Based on a detailed X-ray analysis and the Raman spectra of the initial and hydrated films, it is shown that the plasma-ion modification of the transition and the metallization of vanadium dioxide (at a hydrogen concentration above 10at. \%) are most likely associated with electron-correlation effects amplified by hydrogen implantation and an increase in the free charge carrier density in the material. Meanwhile, the structural changes at the transition practically do not manifest themselves: the hydrated metallic vanadium dioxide remains in the monoclinic phase. The results of plasma-immersion implantation are compared with experiments where other methods of hydration of vanadium dioxide are applied.
\end{abstract}

Keywords: Vanadium dioxide; Metal-Insulator transition; Crystal structure; Doping; Plasma-immersion ion implantation.

\section{Introduction}

The metal-insulator transition (MIT) in $\mathrm{VO}_{2}$, occurring at $\mathrm{Tt}=340 \mathrm{~K}$, is of great importance both from the viewpoint of technical applications and in fundamental terms of understanding the physics of MIT [1,2]. One of the effective ways to change the transition parameters and study its physical mechanism is doping with various elements, including hydrogen [3-10]. Previously we have reported $[11,12]$ on the change in the properties of vanadium dioxide films and in the MIT parameters at hydrogenation by the method of plasma-immersion ion implantation (PIII). It has been found that the introduction of hydrogen by the PIII method into vanadium dioxide films with a relatively low hydrogen concentration (less than 10 at.\%) leads to a decrease in the conductivity jump, i.e. to the suppression of MIT, and above 10 at. $\%$ - to the complete metallization of the $\mathrm{VO}_{2}$ film. As a result of the implantation of hydrogen ions, which play the role of electron donor centers, it is possible to create a metastable $\mathrm{VO}_{2}$ phase with metallic conductivity at a temperature below the temperature of the equilibrium phase transition for the undoped material. In addition, by varying the implantation dose, one can change the conduction jump and the phase transition temperature, which is important for practical applications $[11,12]$.

One of the most important and still unresolved problems in the $\mathrm{VO}_{2}$ metallization with hydrogen is the question of whether such metallic vanadium dioxide remains in the monoclinic phase characteristic of the crystalline state of the material at $\mathrm{T}<\mathrm{Tt}$, or the doping with hydrogen simultaneously leads to the structural phase transition into the rutile tetragonal phase corresponding to metallic pure (undoped) $\mathrm{VO}_{2}$ at $\mathrm{T}>\mathrm{Tt}$.

In some works (see, for example, [3]), it is argued that during the metallization with hydrogen, $\mathrm{VO}_{2}$ does not change the structure, which is recognized as an argument in favor of the correlation-induced Mott mechanism as the main driving force in the development of the MIT in $\mathrm{VO}_{2}$. In other papers [4,6], it is reported that, on the contrary, the introduction of hydrogen results in a structural transition to the rutile phase, and this, in turn, drastically changes the view on the driving force of the MIT and highlights the Peierls structural transition. It should be noted that 
such discrepancies are only natural, if we take into account the complexity of conducting an ideally correct experiment, since there exists a possibility of the hydrogen gradual release from the film during the measurement process.

In the works $[3,4]$, the hydration of $\mathrm{VO}_{2}$ films has been carried out by holding the samples in heated glycerol. The penetration of hydrogen into the vanadium dioxide films occurs from thermally decomposing glycerol. The temperature dependences of the conductivity and reflection spectra of pure and hydrogenated samples, as well as the Raman spectra, have been studied. According to [3], for a hydrogenated film, the Raman spectrum characterizing the monoclinic phase does not depend in any way on the degree of doping, i.e. no structural changes in the crystal lattice occur during hydrogenation, despite the strong changes in the optical constants and electrical conductivity of the film.

On the other hand, the main conclusions reached by the authors of [4] are that, as the degree of hydrogenation increases, the MIT in $\mathrm{H}_{\mathrm{x}} \mathrm{VO}_{2}$ is suppressed and may completely disappear at $\mathrm{x} \sim 0.04$. The instability of hydrated vanadium dioxide does not unequivocally confirm or disprove this assumption. Yet the authors believe that the hydrated $\mathrm{VO}_{2}$ possesses a tetragonal structure.

In the work [6], hydrogen stabilization of metallic vanadium dioxide in single-crystal nanobeams has been studied. Raman and conventional optical microscopy, electron diffraction and transmission electron microscopy provide evidence that the structure of the metallic post-hydrogenation state is similar to that of the rutile state. First-principles electronic structure calculations confirm that a distorted rutile structure is energetically favored following hydrogenation, and also that such doping favors metallicity from both the Mott and Peierls perspectives.

Note that the main methods of hydration of vanadium dioxide are based on thermal diffusion of atomic [7] or molecular [6,8] hydrogen. For example, in [6], the samples were annealed in a tube furnace in the hydrogen flow at $\mathrm{T}=180^{\circ} \mathrm{C}$ for 12 hours. Some other methods, such as cathode polarization in an electrolyte [9], the above-mentioned exposure to hot glycerol $[3,4]$, and even exposure to acid solution (using metal particles to prevent dissolution) [10], have also been applied.

The method of hydration proposed in this work for $\mathrm{VO}_{2}$ using PIII [11-13] is fundamentally new as compared to the above. The PIII method [14], first used in the present work (and in our previous works [11-13]) for doping vanadium oxide thin films, possesses a number of advantages. At the PIII treatment, the sample is placed directly into the low-temperature plasma, and the implantation is carried out immediately over the entire surface of the sample. This leads to a uniform implantation over a large area with a rapid gaining the necessary dose (and, hence, the dopant concentration), while the treatment process is low-temperature. The doping homogeneity depends only on the plasma uniformity above the sample surface, and the energy of the implanted ions can be varied from unities to hundreds of $\mathrm{keV}$ [12].
One of the advantages of the PIII method, exactly for vanadium dioxide, is that, as shown in [13], hydrogen, when a sample is heated or stored after doping, leaves the $\mathrm{VO}_{2}$ films much slower than at doping in hot glycerol. In addition, we note that in a recent paper [15], it has been reported that ion implantation (in this case, in a noble gas plasma) can be used to modify the parameters of the MIT in vanadium dioxide by creating defects.

The aim of this work is to investigate the regularities of modification of the crystal structure and physical properties of vanadium dioxide thin films when they are doped with hydrogen using the method of plasma-immersion ion implantation.

\section{Experimental Methods}

Thin film samples of 200-nm-thick polycrystalline vanadium dioxide on glass-ceramic and single-crystal silicon substrates were fabricated using an AJA ORION 5 setup by reactive magnetron sputtering in a mixture of argon and oxygen (the partial pressures of $\mathrm{Ar}$ and $\mathrm{O}_{2}$ were 4.3 and 0.7 Torr, and the DC generator power was $200 \mathrm{~W}$ ) with subsequent annealing at a temperature of $500^{\circ} \mathrm{C}$ in oxygen.

The implantation of hydrogen into the $\mathrm{VO}_{2}$ films was carried out in the PIII setup described in [12] under the following conditions the discharge current was $9.5 \mathrm{~A}$, discharge voltage $-65.9 \mathrm{~V}$, cathode heating current $-65 \mathrm{~A}$, pressure $-4 \mathrm{~Pa}$, and the gas flow was 0.0018 $\mathrm{m}^{3} \mathrm{~Pa} / \mathrm{s}$. The amplitude of the voltage pulse $\mathrm{V}_{\text {imp }}$ applied to the sample was $2 \mathrm{kV}, \mathrm{I}_{\mathrm{imp}}-20 \mathrm{MA}$; the pulse duration was $\Delta=10 \mu \mathrm{s}$, the time of sample treatment in plasma varied in the range of 1 to 5 minutes, and the pulse repetition rate was $1.5 \mathrm{kHz}$.

The samples were characterized by X-ray diffraction (XRD) analysis and dispersive Raman spectrometry. The XRD analysis was carried out using a DRON-3 diffractometer with $\mathrm{CuK \alpha}$ radiation (wavelength $1.5418 \AA$ ), and a LiF crystal monochromator was installed in the reflected beam. The measurements were conducted over the angular range $2 \theta=10^{\circ}$ to $70^{\circ}$ with the step of $0.1^{\circ}$, and the time of shooting of each point was 25 seconds. Also, we used an ARL X'TRA diffractometer with an HTK 2000 high-temperature chamber to measure XRD patterns in the temperature range 20 to $100{ }^{\circ} \mathrm{C}$. The $\mathrm{CuK} \alpha$ radiation was applied in this case too, and the scattering angular range $2 \theta$ was taken from $24^{\circ}$ to $32^{\circ}$ which corresponded to the $\mathrm{VO}_{2}$ (011) diffraction line. The shooting step was $0.1^{\circ}$, and the shooting time of each point was 2 seconds.

The Raman analysis of the samples, both initial and after hydrogen implantation, was made using a Nicolet Almega XR Dispersive Raman spectrometer with the reference beam of 532$\mathrm{nm}$ in the Raman shift spectral range of $100-1500 \mathrm{~cm}^{-1}$.

\section{Results and Discussion}

The measurements of the conductivity temperature dependences show that at the introduction of hydrogen by the PIII method into the vanadium dioxide films, a small concentration of hydrogen (less than 10 at. \%) leads to a decrease in the conductivity jump, i.e. to the suppression of the MIT [11-13], and above 10\% - 
to the metallization of the film. In this case, the conductivity value $\sigma$ and the dependence $\sigma(\mathrm{T})$ correspond to the metal phase of $\mathrm{VO}_{2}$ in the entire temperature range $\left(20-90^{\circ} \mathrm{C}\right)$, and not only above $\mathrm{Tt}$ $\sim 70{ }^{\circ} \mathrm{C}$ (Figure1). The doping level magnitude has been calculated from the implantation dose [12], and it is rather some estimate, taking into account the possibility of a non-uniform distribution of hydrogen across the film thickness (based on calculations of the implanted impurity concentration profile [13]). However, the obtained value of $\sim 10$ at. \% significantly exceeds the value of 2 at. $\%$ achieved in [4], where the authors argue that the metallization should occur at a hydrogen concentration of $>4$ at. $\%$. Therefore, it can be assumed that metallization occurred as a result of the PIII, which also corresponds to the data of [7] where vanadium dioxide nanobeams have been hydrated in an atomic hydrogen flow at 100 ${ }^{\circ} \mathrm{C}$.

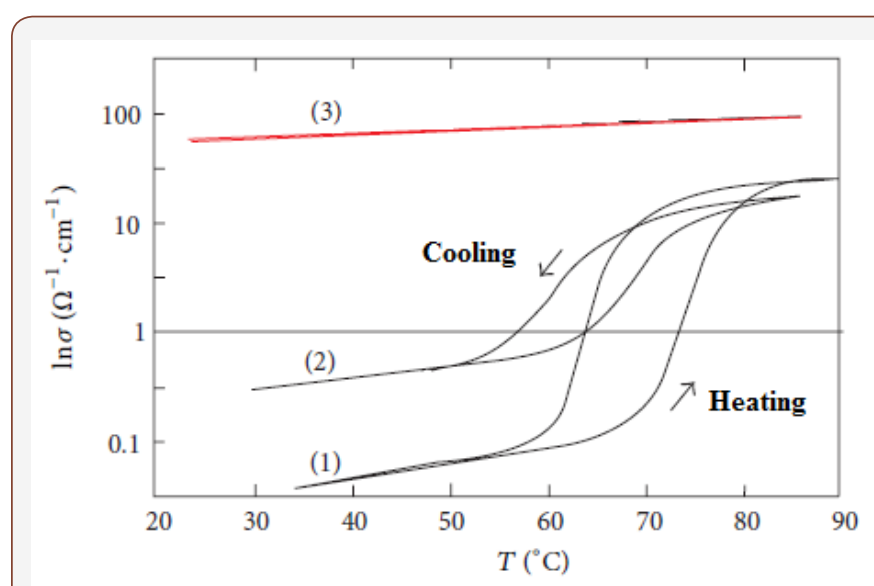

Figure 1: Conductivity temperature dependence of $\mathrm{VO}_{2}$ film: initial sample (1), the region with implanted hydrogen with a low dose, $<7.5 \cdot 10^{16} \mathrm{~cm}^{-2}(2)$, and the region with implanted hydrogen with the dose of $7.5 \cdot 10^{16} \mathrm{~cm}^{-2}(3)[11,12]$.

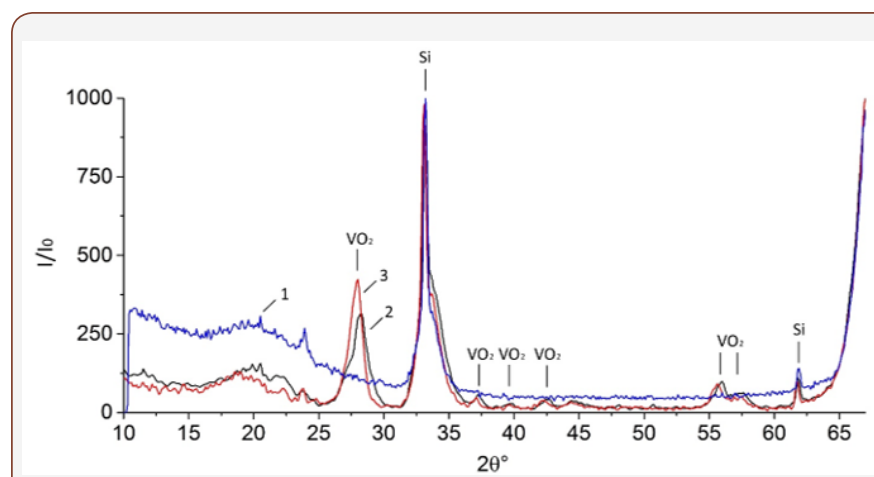

Figure 2: XRD patterns: 1-Si (100) substrate, $2-\mathrm{VO}_{2}$ initial film on silicon, $3-\mathrm{VO}_{2}$ film on silicon after hydrogen implantation implantation dose $-7.5 \cdot 10^{16} \mathrm{~cm}^{-2}$.

For the initial $\mathrm{VO}_{2}$ sample on $\mathrm{Si}$ and for the sample after hydrogen implantation (with the dose of $7.5 \cdot 1016 \mathrm{~cm}^{-2}$, which corresponds to the hydrogen concentration in the film of 10.5 at. $\%[11,12])$, the XRD patterns are obtained (Figure 2). The biggest peak (at $2 \theta=33.12^{\circ}$ ) and the peak at $61.88^{\circ}$ belong to the Si (100) substrate $[16,17]$. The remaining marked peaks correspond to the $\mathrm{VO}_{2}$ film $[17,18]$.
One can reveal the crystal phase of $\mathrm{VO}_{2}$ (monoclinic or tetragonal) by the presence or absence of peaks on the XRD pattern, as well as by their displacement [18]. The peak (102) with an interplanar spacing of $2.68 \AA$ is present on the XRD pattern of the monoclinic phase [18] and absent on the tetragonal one (Figure 3). However, on the obtained diffractograms, this peak is covered with a peak of silicon (Figure 2).

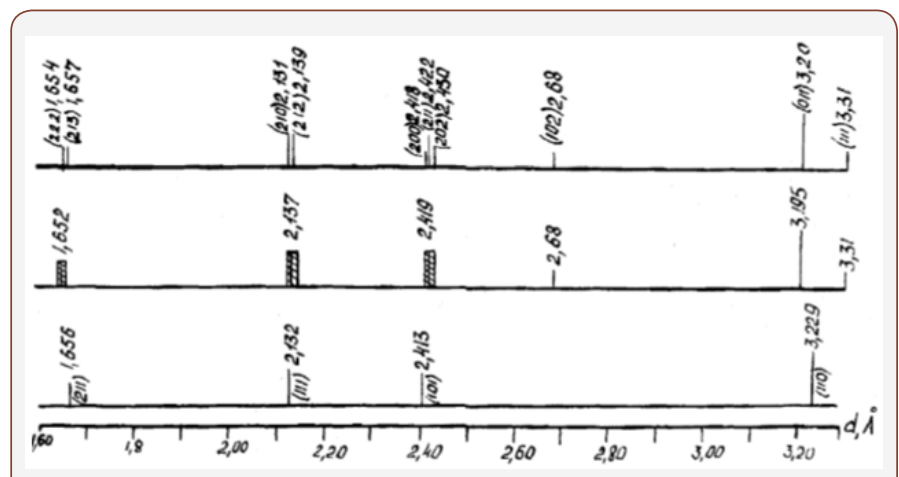

Figure 3: XRD bar graphs of $\mathrm{VO}_{2}$ : upper - theoretical (monoclinic lattice, C4s); middle - low-temperature phase; lower - hightemperature phase (tetragonal lattice, rutile C4) [18]

Also, one can judge about the $\mathrm{VO}_{2}$ crystal phase by the position of the peak (011) [18]. Therefore next, we have carried out additional experiments with a high-temperature chamber. Moreover, the measurements have been conducted not over the entire overview XRD pattern, but specifically for the line (011).

Table 1: Interplanar spacings of $\mathrm{VO}_{2}$ film before and after hydrogen implantation at various temperatures.

\begin{tabular}{|c|c|c|}
\hline & Initial sample & After H implantation \\
\hline $\mathrm{T}, \stackrel{\circ}{ } \mathrm{C}$ & $\mathrm{d}, \AA$ & $\mathrm{d}, \AA$ \\
\hline 25 & 3.154 & 3.155 \\
\hline 48 & 3.159 & 3.166 \\
\hline 58 & 3.173 & 3.168 \\
\hline 73 & 3.172 & 3.177 \\
\hline 83 & 3.175 & 3.176 \\
\hline 93 & 3.179 & 3.176 \\
\hline
\end{tabular}

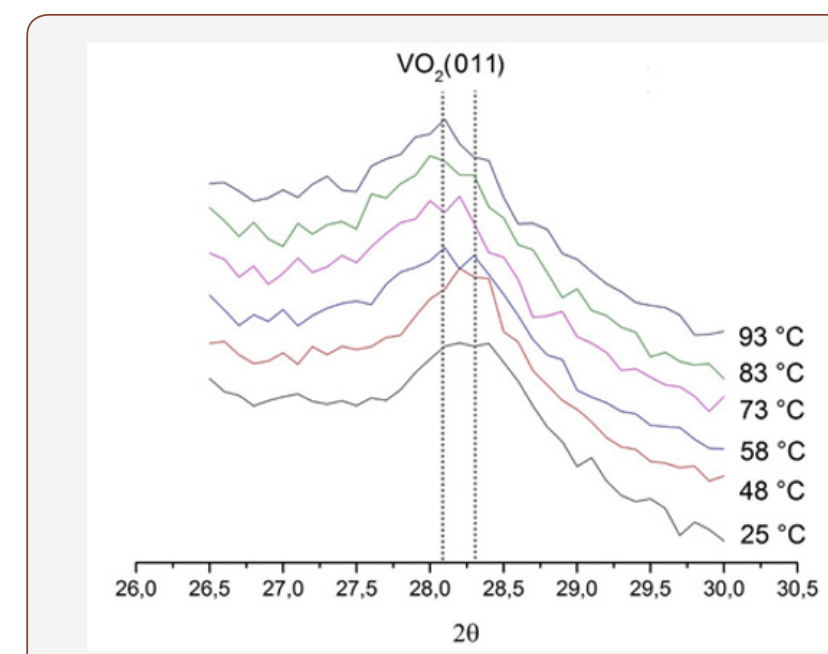

Figure 4: Diffraction line (011) of the initial $\mathrm{VO}_{2}$ film sample at different temperatures. 


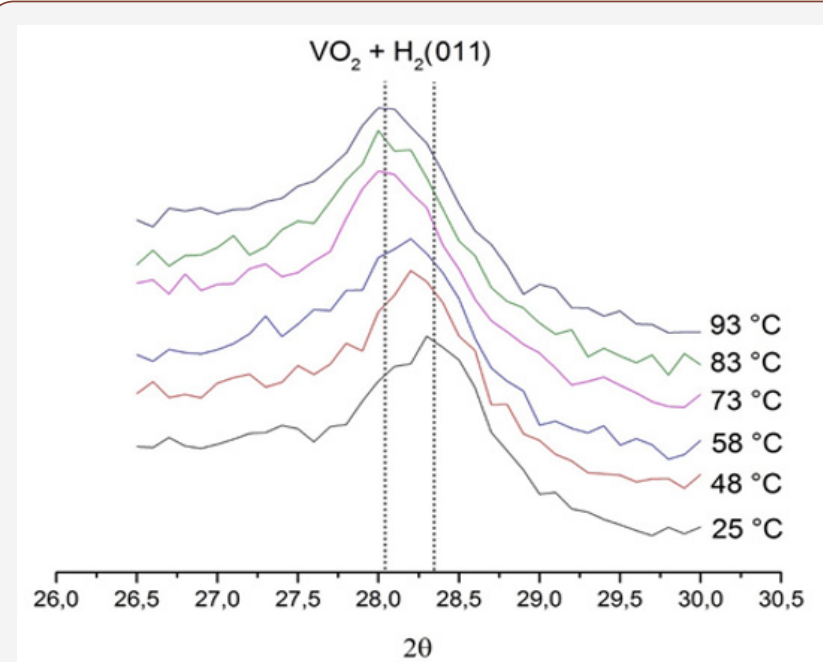

Figure 5: Diffraction line (011) of the $\mathrm{VO}_{2}$ film sample at different temperatures after hydrogen implantation with the dose of $7.5 \cdot 10^{16} \mathrm{~cm}^{-2}$

Figures 4 \& 5 show the profiles of the diffraction line corresponding to the (011) $\mathrm{VO}_{2}$ peak at various temperatures for the initial film and the film after hydrogen implantation. Table 1 presents the interplanar spacings.

From Table 1 it can be seen that the line (011) position almost does not vary after implantation, and its shift occurs only when the sample is heated. Moreover, this shift is almost the same for both the original film and the film after hydrogen implantation. Therefore, we arrive at the conclusion that in both the samples at room temperature $\mathrm{VO}_{2}$ has the monoclinic structure, and when

heated, a transition to the tetragonal phase occurs. Moreover, the structural transition temperature for both the films remains identical, approximately $70 \stackrel{\circ}{\circ}$.

For more information on the structure of the $\mathrm{VO}_{2}$ sample after hydrogenation, the Raman spectra of the films on silicon have been obtained. Figure 6 shows the Raman spectra of the initial and hydrogenated $\mathrm{VO}_{2}$ films, and the recorded peaks are presented in Table 2 in comparison with the literature data [19]. The peaks at $521 \mathrm{~cm}^{-1}$ and $949 \mathrm{~cm}^{-1}$ belong to the Si substrate. It can be seen from Figure 6 that the spectra of the initial and hydrogenated films practically coincide and correspond to the spectrum of crystalline monoclinic $\mathrm{VO}_{2}$.

Table 2: Raman peaks of $\mathrm{VO}_{2}$.

\begin{tabular}{|c|c|}
\hline Data from [19] & $\mathrm{VO}_{2}$ Film Under Study \\
\hline \multicolumn{2}{|c|}{ Wavenumber $\left(\mathrm{cm}^{-1}\right)$} \\
\hline 392 & 387 \\
\hline 395 & - \\
\hline 444 & 439 \\
\hline 453 & - \\
\hline 489 & - \\
\hline 503 & 495 \\
\hline- & 521 \\
\hline 585 & - \\
\hline 618 & 615 \\
\hline 650 & - \\
\hline 825 & 820 \\
\hline- & 949 \\
\hline
\end{tabular}

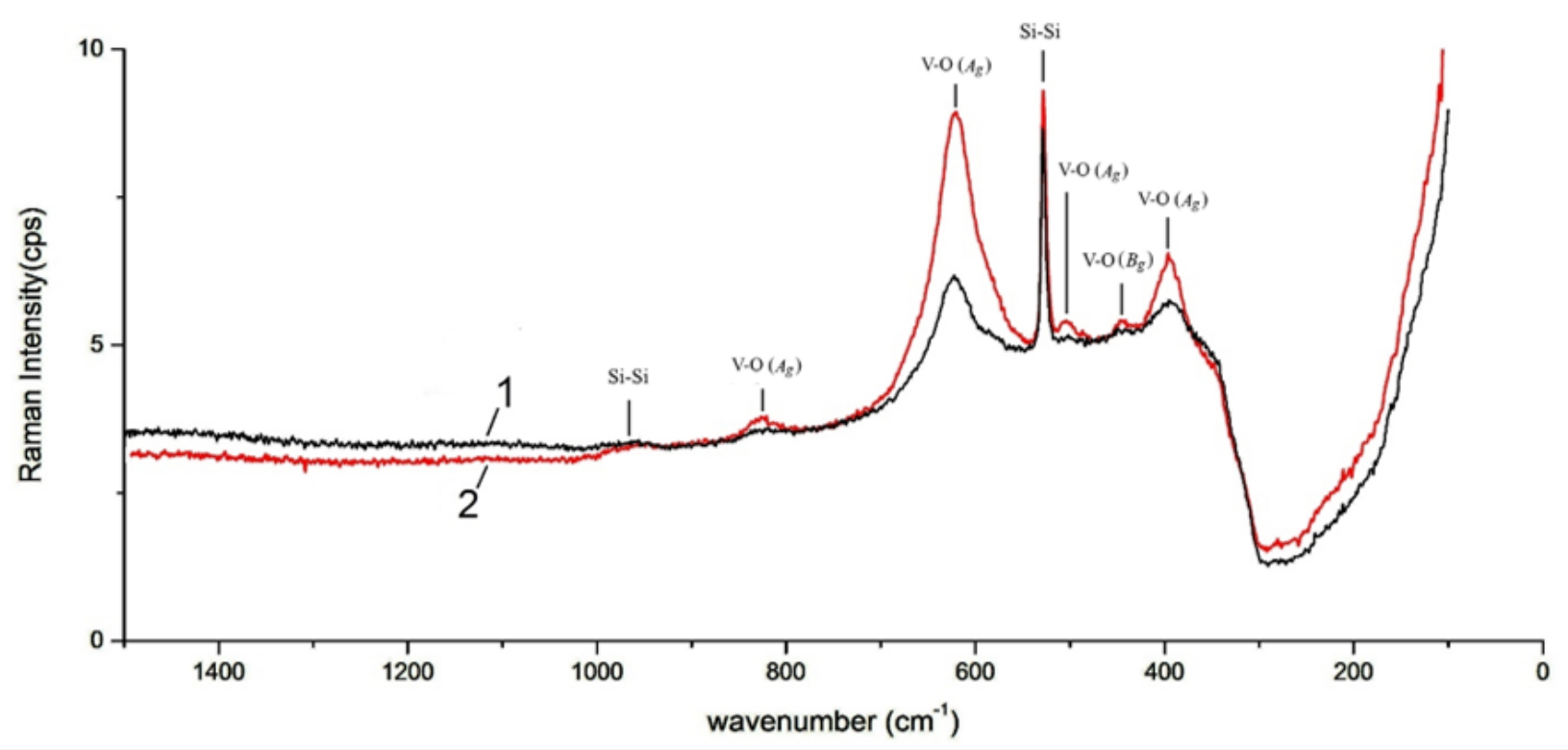

Figure 6: Raman spectra of $\mathrm{VO}_{2}$. 1-initial film, 2-hydrated film, implantation dose is $7.5 \cdot 10^{16} \mathrm{~cm}^{2}$.

This result is consistent with the results of [3]. Thus, when doping with hydrogen to a level of 10.5 at. \%, vanadium dioxide transits into the metal phase, while the monoclinic crystal structure, corresponding to the low-temperature semiconductor phase of pure $\mathrm{VO}_{2}$, is preserved. Recall that, as shown above, the preservation of the monoclinic structure at hydrogenation is also confirmed by the XRD data.

The results obtained indicate the electron-correlation mechanism of MIT (Mott transition) in vanadium dioxide. As is 
noted in [3], such a transition to a new "metallic" monoclinic state is the Mott electronic transition, its onset precedes the structural phase transition, and the Mott transition itself initiates a structural phase transition at $\mathrm{Tt}=68^{\circ} \mathrm{C}$.

However, it should be noted that a number of works (see, e.g., $[4,6])$ report on the observation of the metallization of vanadium dioxide at hydrogenation, accompanied by a structural transition from the monoclinic phase to the tetragonal one. Moreover, in [20], the authors report the results of density functional calculations showing that hydrogen-induced lattice distortion stabilizes the metallic phase. They also show that hydrogen doping is energetically favored, and structural (not electronic) changes play a leading role in the transition mechanism: this mechanism is thus, in essence, a Peierls structural transition (according to [20]). Meanwhile, merely the formation of the tetragonal structure at hydrogenation is not an evidence of the impossibility of the Mott transition, since, in this case, the Mott electronic transition, caused by an increase in the density of free carriers (electrons), might initiate a structural phase transition at $\mathrm{T}<68{ }^{\circ} \mathrm{C}$ with a change in the lattice symmetry from monoclinic to tetragonal $[3,21,22]$.

Therefore, the monoclinic metal phase in hydrogenated $\mathrm{VO}_{2}$, which is observed in the present work (as well as in [3]), is apparently metastable. It is similar to the intermediate (Figure 7) metastable phase previously observed in other experiments when this non-equilibrium monoclinic metal phase is formed as a result of laser irradiation $[1,23,24]$, under pressure [25], at chemisorption of organic molecules [26] or in heterostructures with nonstoichiometric (oxygen-deficient) $\mathrm{VO}_{2}$ [27]. The question of why, when doping with PIII (as well as when processing in glycerol under certain conditions [3]), the metastable monoclinic metal phase is formed, while other methods of hydrogenation result in the tetragonal phase, requires additional study.

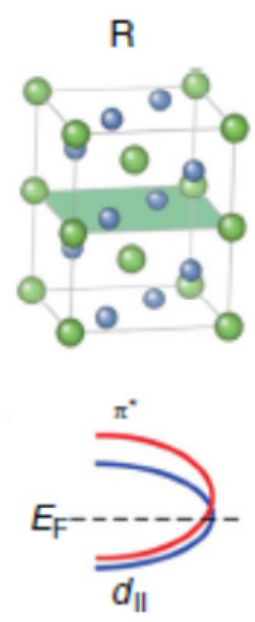

Metal

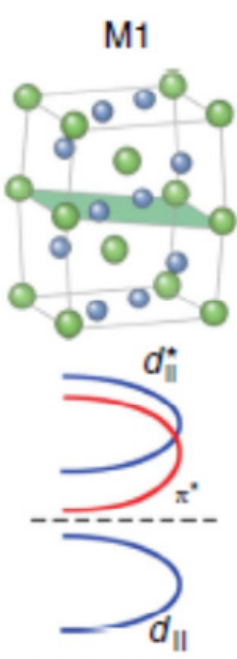

Insulator

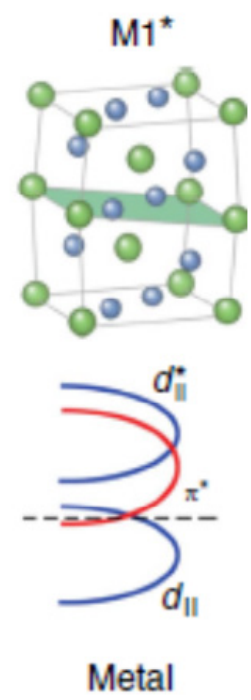

Metal
Figure 7: Crystal structures of different phases of $\mathrm{VO}_{2}$ and corresponding electronic band structures [26]. Left: metal tetragonal rutile phase $(\mathrm{R})$; middle: insulating monoclinic phase (M1); right: intermediate metal-like monoclinic $\left(\mathrm{M} 1^{*}\right)$ phase. Proposed band diagrams are adapted from [23].
Summarizing, we once again emphasize that when doping by PIII with hydrogen to a level of more than 10 at. \%, vanadium dioxide passes into the metal phase, yet the monoclinic crystal structure is preserved. Thus, structural changes are not a necessary condition for the semiconductor-to-metal transition to occur, which indicates the electron-correlation nature of the MIT (in concordance with the Mott transition mechanism) in vanadium dioxide. Note that we are talking only about a simplified description of the MIT nature in $\mathrm{VO}_{2}$, i.e. which of the two mechanisms, structural or electronic, prevails. Therefore, surely, the contribution of the crystal structure and its changes during the introduction of hydrogen cannot be completely denied. As is pointed out in the survey [2]: "Recent theoretical treatments tend to bridge the gap between the purely Mott-like and purely Peierls-like pictures. Cluster-DMFT simulations have presented a so-called "correlation assisted Peierls transition" scenario, concluding that electronic correlations are necessary in leading the bandgap opening process". Furthermore, as shown in the recent paper [22], the correlation effects make a significant contribution to the electronic structure of all the three stable phases (M1, M2 and R [2]) of vanadium dioxide.

\section{Conclusion}

When doping with hydrogen by the PIII method, vanadium dioxide remains in the monoclinic phase up to the level of its metallization (more than 10 at. \% of hydrogen). This is evidenced by the analysis of the temperature dependence of the (001) XRD peak position of the initial and hydrated films. This position, corresponding to the interplanar spacing $d=3.15 \AA$, does not depend on the implantation of hydrogen and in both cases shifts with increasing temperature almost identically, indicating a structural transition to the tetragonal phase at $\mathrm{Tt} \sim 70^{\circ} \mathrm{C}$. Also, the Raman spectra of the initial film of vanadium dioxide and the hydrated film above the metallization threshold are identical and correspond to the monoclinic phase.

Thus, structural changes are not a necessary condition for the semiconductor-to-metal transition, which indicates the electroncorrelation nature of the MIT (in concordance with the Mott transition mechanism) in vanadium dioxide. This conclusion is also confirmed by the results presented in numerous other works, in which the possibility of the existence of the $\mathrm{VO}_{2}$ metastable metal phase with monoclinic symmetry of the crystal lattice is shown.

\section{Acknowledgements}

This work was supported by the RF Ministry of Education and Science, project no. 16.5857.2017/8.9 (state program).

\section{Conflict of Interest}

The authors declare that they have no conflicts of interest.

\section{References}

1. A A Bugaev, B P Zakharchenya, F A Chudnovskii (1979) Metalsemiconductor phase transition and its applications. Nauka, Leningrad, Russia, p.184.

2. K Liu, S Lee, Sh Yang, O Delaire, J Wu (2018) Recent progresses on physics and applications of vanadium dioxide. Materials Today 21(8): 875-896. 
3. AV Ilinskiy, O E Kvashenkina, E B Shadrin (2001) Protonic metallization of the monoclinic phase in $\mathrm{VO}_{2}$ films. Semiconductors 45: 1153-1157.

4. VN Andreev, VA Klimov, ME Kompan (2012) Influence of hydrogenation on electrical conductivity of vanadium dioxide thin films. Phys Solid State 54(3): 601-606.

5. AV Ilinskiy, VM Kapralova, EB Shadrin (2008) Metal-semiconductor phase transition in hydrated films of vanadium dioxide. St. Petersburg Polytechnic University Journal - Physics and Mathematics 6: 103-109.

6. J Wei, H Ji, W Guo, A H Nevidomskyy, D Natelson (2012) Hydrogen stabilization of metallic vanadium dioxide in single crystal nanobeams. Nature Nanotechnology 7: 357-362.

7. J Lin, H Ji, M W Swift, W J Hardy, Z Peng, et al. (2014) Hydrogen diffusion and stabilization in single-crystal $\mathrm{VO}_{2}$ micro/nanobeams by direct atomic hydrogenation. Nano Lett 14(9): 5445-5451.

8. W J Hardy, H Ji, H Paik, D G Schlom, D Natelson (2017) Mesoscopic quantum effects in a bad metal, hydrogen-doped vanadium dioxide. J Phys Condens Matter 29(18): 185601.

9. H Yoon, M Choi, T Lim, H Kwon, K Ihm, et al. (2016) Reversible phase modulation and hydrogen storage in multivalent $\mathrm{VO}_{2}$ epitaxial thin films. Nature Materials 15: 1113-1119.

10. Y Chen, Z Wang, S Chen, H Ren, L Wang, et al. (2018) Non-catalytic hydrogenation of VO2 in acid Solution. Nature Communications 9(818): 8.

11. S V Burdyukh, G B Stefanovich, A L Pergament, O Y Berezina, N A, Avdeev, et al. (2016) Modification of the properties of vanadium dioxide by plasma-immersion ion implantation. Technical Physics Letters 42(1): 32-35.

12. S Burdyukh, O Berezina, A Pergament (2018) Modification of the properties of vanadium oxide thin films by plasma-immersion ion implantation. Advances in Condensed Matter Physics 2018: 9789370.

13. SV Burdyukh (2018) Modification of the properties of vanadium oxides by the method of plasma-immersion ion implantation. $\mathrm{PhD}$ thesis, Petrozavodsk: PetrSU.

14. PK Chu, S Qin, C Chan, NW Cheung, L A Larson (1996) Plasma immersion ion implantation-a fledging technique for semiconductor processing. Mater Sci Eng R 17(6-7): 207-280.
15. Q Jia, J Grenzer, H He, W Anwand, Y Ji, et al. (2018) 3D local manipulation of the metal-insulator transition behavior in $\mathrm{VO}_{2}$ Thin film by defectinduced lattice engineering. Adv Mater Interfaces 5(8): 9.

16. JS Kasper, SM Richards (1964) The crystal structures of new forms of silicon and germanium. Acta Crystallographica 17: 752-755.

17. RW G Wyckoff (1963) Crystal structures. New York: Interscience Publishers 65(9): 467.

18. AS Oleinik (2004) Vanadium dioxide films in devices of indicator technology and microelectronics. Doctor of Sci Dissertation Saratov.

19. M Pan, J Liu, H Zhong, S Wang, Z Li, et al. (2004) Raman study of phase transition in $\mathrm{VO}_{2}$ thin films. Journal of Crystal Growth 268(1-2) 178-183.

20. KH Warnick, B Wang, ST Pantelides (2014) Hydrogen dynamics and metallic phase stabilization in $\mathrm{VO}_{2}$. Appl Phys Lett 104: 101913.

21. A Pergament (2003) Metal-insulator transition: The Mott criterion and coherence length. Journal of Physics Condensed Matter 15(19): 32173223 .

22. TJ Huffman, C Hendriks, EJ Walter, Y Joonseok, J Honglyoul, et al. (2017) Insulating phases of vanadium dioxide are Mott-Hubbard insulators. Physical Review B 95(7): 075125.

23. HT Kim, YW Lee, BJ Kim, BG Chae, SJ Yun, et al. (2006) Monoclinic and correlated metal phase in $\mathrm{VO}_{2}$ as evidence of the Mott transition: coherent phonon analysis. Phys Rev Lett 97(26): 266401.

24.VR Morrison, RP Chatelain, KL Tiwari, A Hendaoui, A Bruhács, et al. (2014) A photoinduced metal-like phase of monoclinic $\mathrm{VO}_{2}$ revealed by ultrafast electron diffraction. Science 346(6208): 445-448.

25. E Arcangeletti, L Baldassarre, D Di Castro, S Lupi, L Malavasi, et al. (2007) Evidence of a pressure-induced metallization process in monoclinic $\mathrm{VO}_{2}$ Phys Rev Lett 98(19): 196406.

26. Z Li, J Wu, Z Hu, Y Lin, Q Chen, et al. (2017) Imaging metal-like monoclinic phase stabilized by surface coordination effect in vanadium dioxide nanobeam. Nature Communications 8: 15561.

27. D Lee, B Chung, Y Shi, G Y Kim, N Campbell, et al. (2018) Isostructural metal-insulator transition in $\mathrm{VO}_{2}$. Science 362(6418): 1037-1040. 